\title{
Adaptive Noise Filter for Real-Time Stress ECG Signal Analysis
}

\author{
Hoang Van Manh, Pham Manh Thang* \\ University of Engineering and Technology, Vietnam National University, Hanoi, Vietnam \\ No. 144, Xuan Thuy, Cau Giay, Hanoi, Vietnam \\ Received: October 27, 2020; Accepted: November 12, 2020
}

\begin{abstract}
The stress Electrocardiogram (ECG) gives more efficient results for the diagnosis of cardiovascular diseases, which may not be apparent when the patients are at rest. However, the noise produced by the movement of the patient and the environment often contaminates the ECG signal. Motion artifact is the most prevalent and difficult type of interference to filter in stress test ECG. It corrupts the quality of the desired signal thus reducing the reliability of the stress test. In this work, we first describe a quantitative study of adaptive filtering for processing the stress ECG signals. The proposed method uses the motion information obtained from a 3-axis accelerometer as a noise reference signal for the adaptive filter and the optimal weight of the adaptive filter is adjusted by the Modified Error Data Normalized Step-Size (MEDNSS) algorithm. Finally, the performance of the proposed algorithm is tested on the stress ECG signal from the subject.
\end{abstract}

Keywords: stress ECG, adaptive filter, motion artifacts, accelerometer.

\section{Introduction}

Stress ECG is widely utilized to assess the performance of heart muscle in terms of the ECG changes. In this test, the maximum workload, the blood pressure, heart respiratory rate as well as the recovery period of repolarization are appreciated. The patient should jog on a treadmill or perform exercises with the difficulty level gradually increased until the patient feels exhausted and stopped. During stress testing, especially in the high-intensity exercise phase, artifacts caused by patient movement can affect the quality of the ECG signal, making it difficult to analyze and diagnose cardiovascular diseases. Motion artifact is often the most difficult type of interference to remove from the ECG signal since its spectrum can completely overlap with the useful ECG signal and its morphology is usually similar to the $\mathrm{P}$, the QRS complex, and the $\mathrm{T}$ waves [1]. From a clinical perspective, motion artifact can lead to misdiagnosis and inappropriate treatment decisions. The efficient removal of disturbances due to patient movement is still an unsolved problem in the clinical setting. In determining heart rate variability, motion artifact is still referred to as an existing problem [2]. Since this type of noise is in-band with ECG and related signals are dynamically changing, it is the most efficient to use the adaptive filter for stress ECG signals.

Several works have been implemented to eliminate artifacts from ECG signals by various filtering algorithms based on Least Mean Squares
(LMS) algorithm. Shafa-at Ali Sheikh et al. [3] proposed a method to remove BLW from ECG signals using State Space Recursive Least Squares (SSRLS) adaptive filter tuned by estimating the frequency BLW in range of $0.15 \sim 0.30 \mathrm{~Hz}$. The tracking of BLW by SSRLS has been done by using a sinusoidal model in state space. Yan Liu et al. [4] proposed an algorithm to measure non-invasively skin strain using a lightemitting diode (LED) and an optical sensor incorporated in an ECG electrode. The results obtained illustrate that the proposed method can effectively eliminate motion artifacts from the stress ECG signal. To eliminate the effect of baseline wander (BLW) on useful ECG signals obtained from a Holter ECG monitor, K. Pandey [5] added an absolute power spectrum of 3-axis accelerometer output connected to an ECG electrode as the noise reference signal for an adaptive filter. The performance of the technique is quantitatively evaluating the signals acquired from several subjects during the post-exercise test. Yoon et al. [6] proposed an algorithm to reduce the motion artifacts by using a 3 -axis accelerometer in the e-textile ECG measurement system. In the experiment, the 3axis accelerometer sensor module ACH-04-08-05 installed in the ECG recording device is used to acquire moment information in three orthogonal directions when the patient is active. These signals are utilized as the noise reference signal of the adaptive filter. Finally, to improve the correlation between the interference signal and the acceleration signal obtained, the normalized LMS (NLMS) algorithm is

\footnotetext{
${ }^{*}$ Corresponding author: Tel.: (+84) 946.863.686

Email: thangpm@vnu.edu.vn
} 
used to optimize the filter parameters. To reduce the impact of motion artifact on the quality of ECG recordings, Tong et al. [7] proposed an adaptive filtering technique for electrode motion artifacts caused by relative motion between the subject's skin and the electrode. In the study, the authors designed two motion sensors to monitor the moment of the electrode, and those two sensors are two-axis anisotropic magnetoresistive (AMR) sensor and threeaxis accelerometers (ACC) respectively. The LMS algorithm is utilized to adjust the optimal weight of the adaptive filter. In the work of Mary Anne D. Raya et al. [8], they used the ADXL105, a single-axis accelerometer manufactured by Analog Devices, to acquire the noise reference signal generated during the patient walking or running on the bicycle ergometer for the adaptive filter. Finally, the two most widely used adaptive filtering algorithms, LMS and Recursive Least Squares (RLS) are utilized to minimize the error signal and suppress motion artifact.

In this study, we use acceleration signals from a 3 -axis accelerometer as the reference input of the adaptive filter, and the optimal weight of the adaptive filter is adjusted by the Modified Error Data Normalized Step-Size (MEDNSS) algorithm to remove motion artifacts. The algorithm is applied to stress ECG and it is shown that the proposed algorithm produces good results.

\section{Materials and methods}

\subsection{Materials}

The block diagram of the adaptive noise canceller using the EDNSS algorithm is shown in Fig.1. According to this diagram, the primary input $d(n)$ consists of the sum of the desired signal $s(n)$ and the uncorrelated noise $n_{0}(n)$. The noise $n_{0}(n)$ is uncorrelated with $s(n)$. The reference input $x(n)$ is another noise $n_{1}(n)$ which is correlated with $n_{0}$ and uncorrelated with $s(n)$. The EDNSS algorithm is a type of normalized Least Mean Square (NLMS) algorithm, in which step size is adjusted from one iteration to the next iteration in weight vector along with the input. The input error tracks on normalizing along with the recent input data. This algorithm overcomes the fixed step size problem in the basic LMS algorithm.

The output and error equations of the EDNSS algorithms are,

$$
\begin{aligned}
& y(n)=w(n) x^{T}(n) \\
& e(n)=d(n)-y(n)
\end{aligned}
$$

where $w$ is the adaptive filter vector weight.

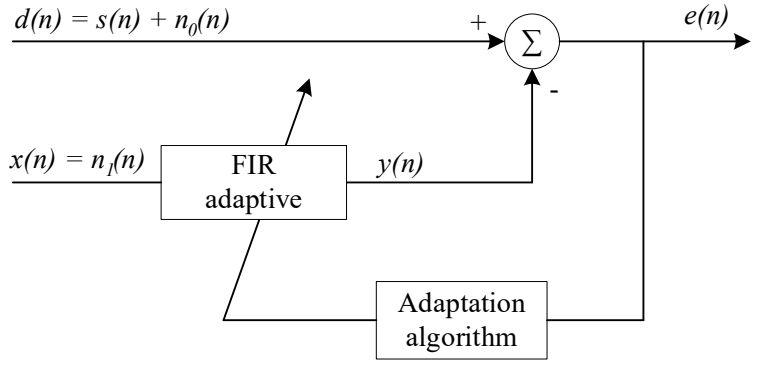

Fig. 1. Adaptive noise canceller using EDNSS algorithm.

The weight equation of the EDNSS algorithms is given by,

$$
\begin{gathered}
w(n+1)=w(n) \\
+\frac{\mu}{\alpha e_{L}(n)^{2}+(1-\alpha) x(n)^{2}} x(n) e(n)
\end{gathered}
$$

where,

$$
\begin{gathered}
x(n)^{2}=x(n) x^{T}(n) \\
e_{L}(n)^{2}=\sum_{i=0}^{L-1}|e(n-i)|^{2}
\end{gathered}
$$

where $n$ represents the total number of samples and $L$ represents a fixed number of samples selected on the basis to get an optimum response. The parameters $\alpha$ and $\mu$ are also chosen in such a manner that the best rate of convergence and the low mean square error are obtained.

\subsection{Methods}

We know that the value $e_{L}(n)^{2}$ is squared norm of error vector for $L$ number of fixed samples which is very large at the beginning of the adaption and rapidly decreases as $n$ increases during adaption [9].

The proposed algorithm may be considered as a modified EDNSS algorithm in which the error vector for fixed samples $L$ is replaced with total samples $n$ then $e_{L}(n)^{2}$ is replaced with $e(n)^{2}$ resulting in increased stability and initial convergence. The effect can be observed in the results.

Now the weight equation of modified EDNSS algorithm is given by

$$
\begin{gathered}
w(n+1)=w(n) \\
+\frac{\mu}{\alpha e(n)^{2}+(1-\alpha) x(n)^{2}} x(n) e(n)
\end{gathered}
$$

where $n$ represents the total number of signal samples, 


$$
e(n)^{2}=\sum_{i=0}^{n-1}|e(n-i)|^{2}
$$

Equation (6) is similar to the original NLMS algorithm and step size is given by

$$
\mu(n)=\frac{\mu}{\alpha e(n)^{2}+(1-\alpha) x(n)^{2}}
$$

where $\mu$ is the initial value of step size.

\section{Results and discussions}

\subsection{Learning curve}

The learning curve is used to assess the performance of an adaptive filter including convergence speed, steady-state error, and stability. A learning curve is a plot of the Mean Square Error (MSE) of the adaptive filter versus time or iteration.

The learning curve for filter order $5, \alpha=0.7$ and $\mu=[0.02,0.1,1]$ is computed. It is observed that as the iteration increases, the adaptive filter MSE remains almost the same and is not converging for high values of $\mu$. The learning curve starts converging only with the smaller value of $\mu$. Fig. 2 shows that the MSE for filter $\operatorname{order} N=5, \alpha=0.7$, and $\mu=0.1$ and 1 are not converging, whereas, for $\mu=0.02$, the MSE reaches the minimum at the end of iteration with the optimal weight.

\subsection{Simulation results}

As discussed earlier, the obtained performance of the proposed algorithm is the most efficient when the parameter $\mu$ is chosen as $0.02, \alpha$ is 0.7 and the filter order is 5 . The proposed algorithm's performance will be tested on the ECG stress test signals of the Glasgow University Database (GUDB) [10]. This database includes $125 \mathrm{ECG}$ signal records collected from 25 subjects with five different tasks (sitting, math test, hand-bike, walking, and jogging on a treadmill). And we utilize the adaptive filter algorithm to reduce motion artifacts. The tap weight is adaptively adjusted by the MEDNSS algorithm. The obtained results are represented in Fig.3, Fig.4, and Fig.5, respectively. Fig. 3 shows the performance of the adaptive filter under the sitting state. Fig.4 depicts the performance of the adaptive filter under jogging on the treadmill at $8 \mathrm{kph}$. Meanwhile, Fig. 5 compares the EMSE values obtained from the EDNSS and MEDNSS algorithms.

It can be seen from Fig.3(b) that the acceleration signal collected by the 3 -axis accelerometer is relatively stable, and there is no large jitter and sudden change. The result obtained by applying the MEDNSS algorithm to the suppression of motion artifacts of ECG signals is shown in Fig.3(c).

Fig.4(b) shows that the 3-accelerometer has obvious jitter, which makes it difficult to detect and locate the $R$ peak. However, there is a correlation between the motion artifact noise of the stress ECG signal and the human motion. Fig.4(c) represents the result of the proposed method in the motion state after filtering.

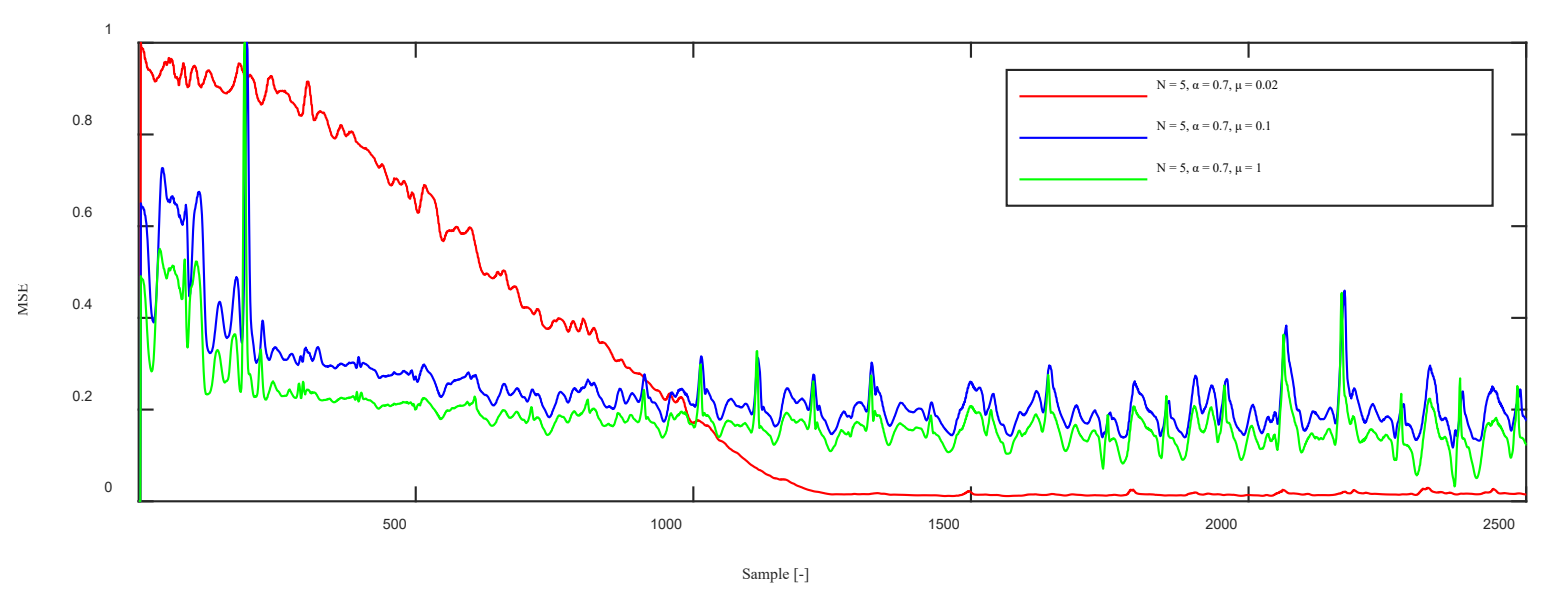

Fig. 2. Learning curves for the proposed filtering technique. 


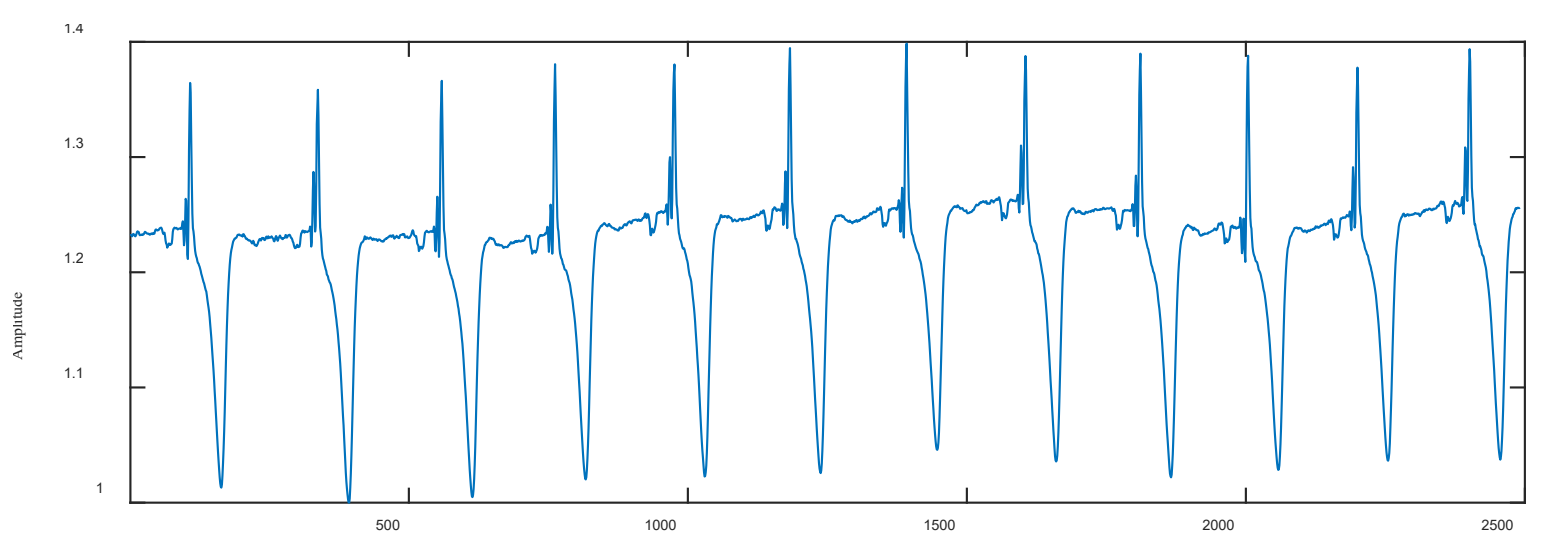

(a) - the real recorded ECG

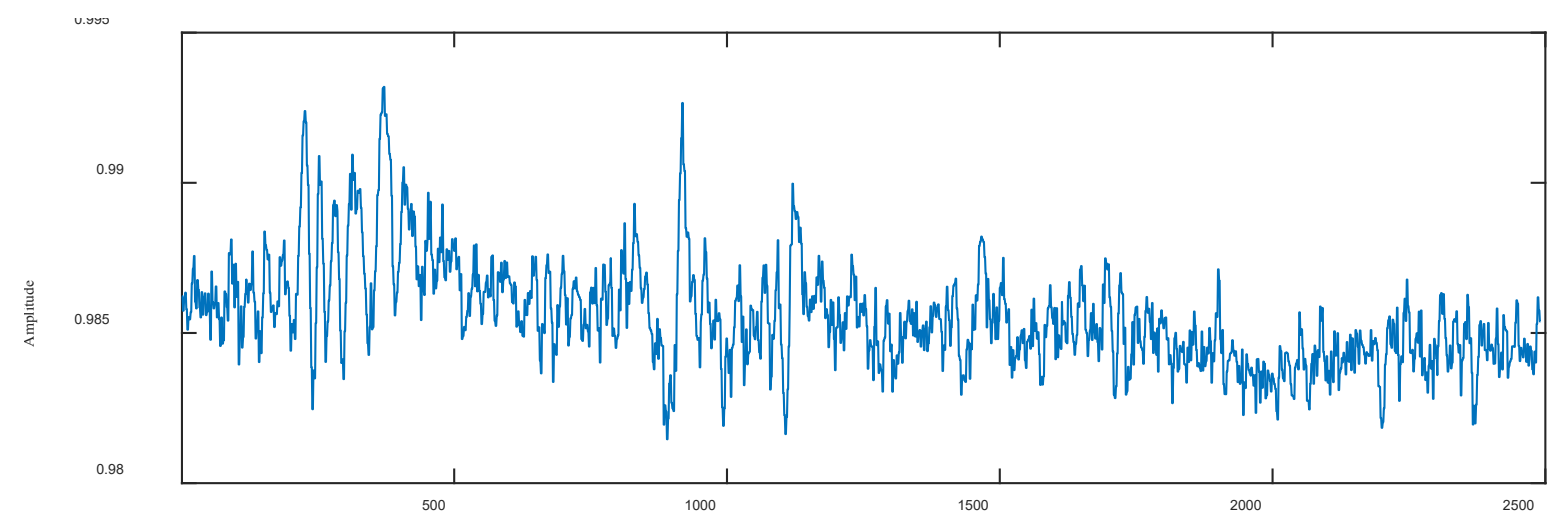

(b) - the reference signal vector

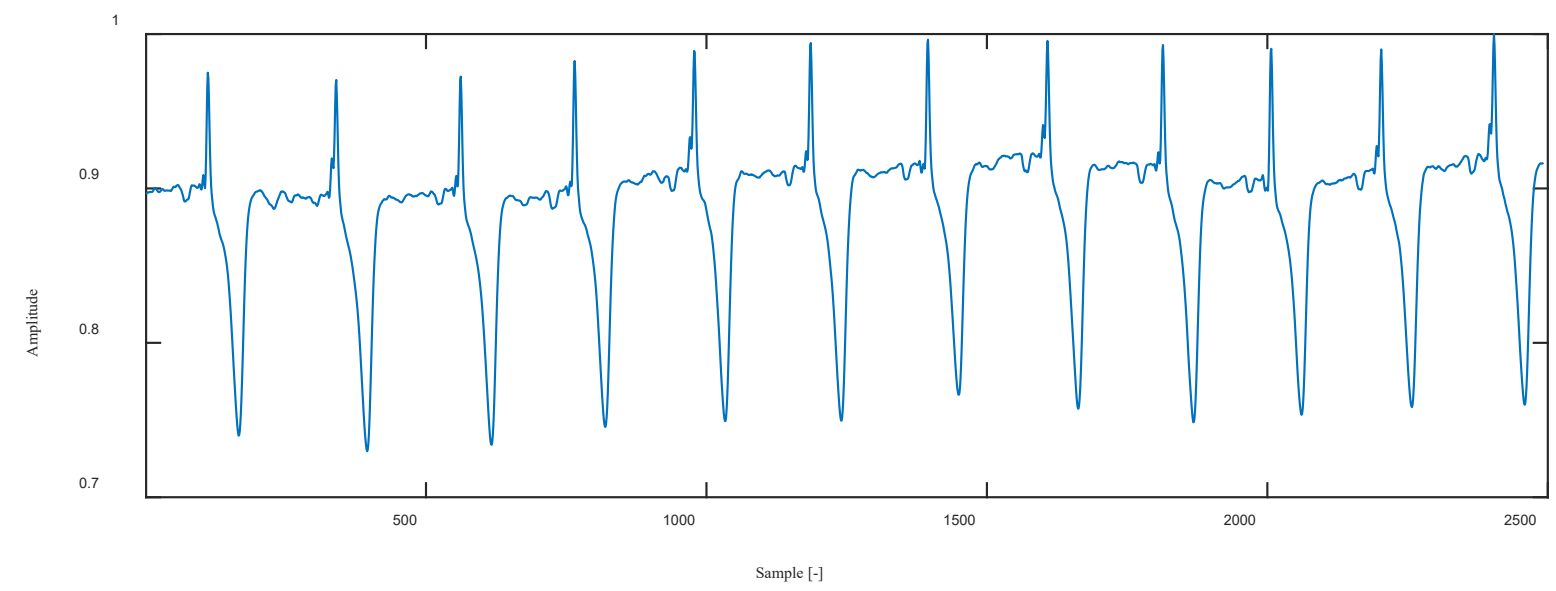

(c) - the filtered ECG by the proposed algorithm

Fig. 3. The performance of the adaptive filter under sitting state: (a) - the real recorded ECG, (b) - the reference signal vector from the accelerometer, and (c) - the filtered ECG by using MEDNSS algorithm. 


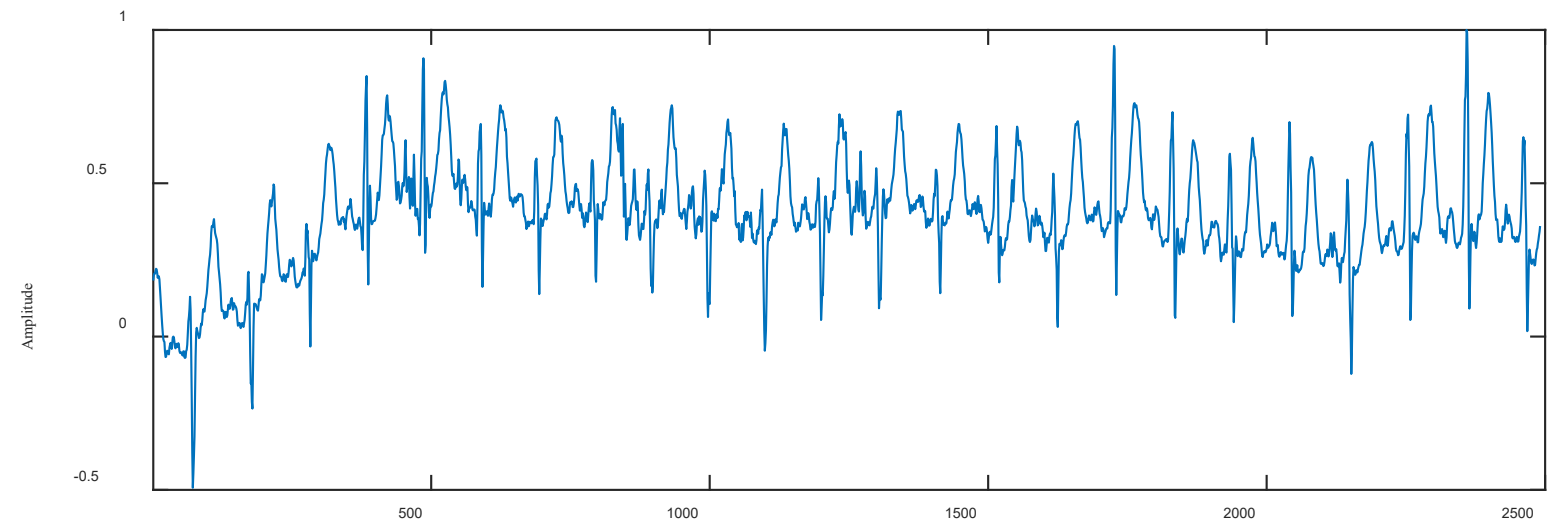

(a) - the real recorded ECG

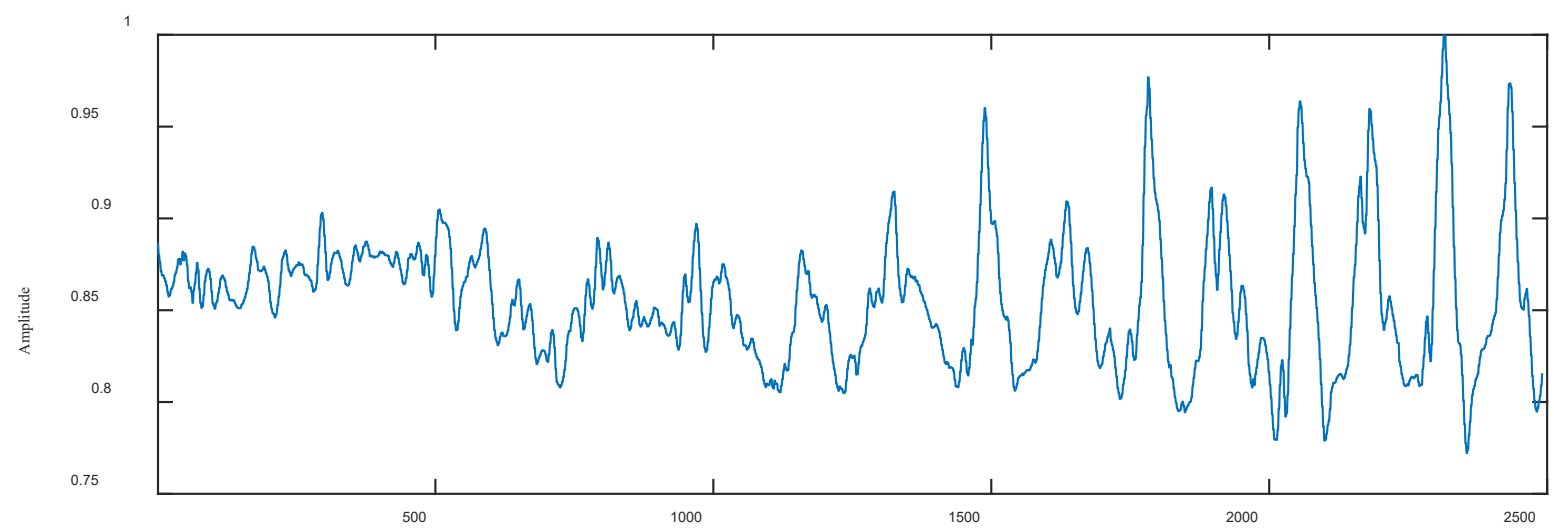

(b) - the reference signal vector

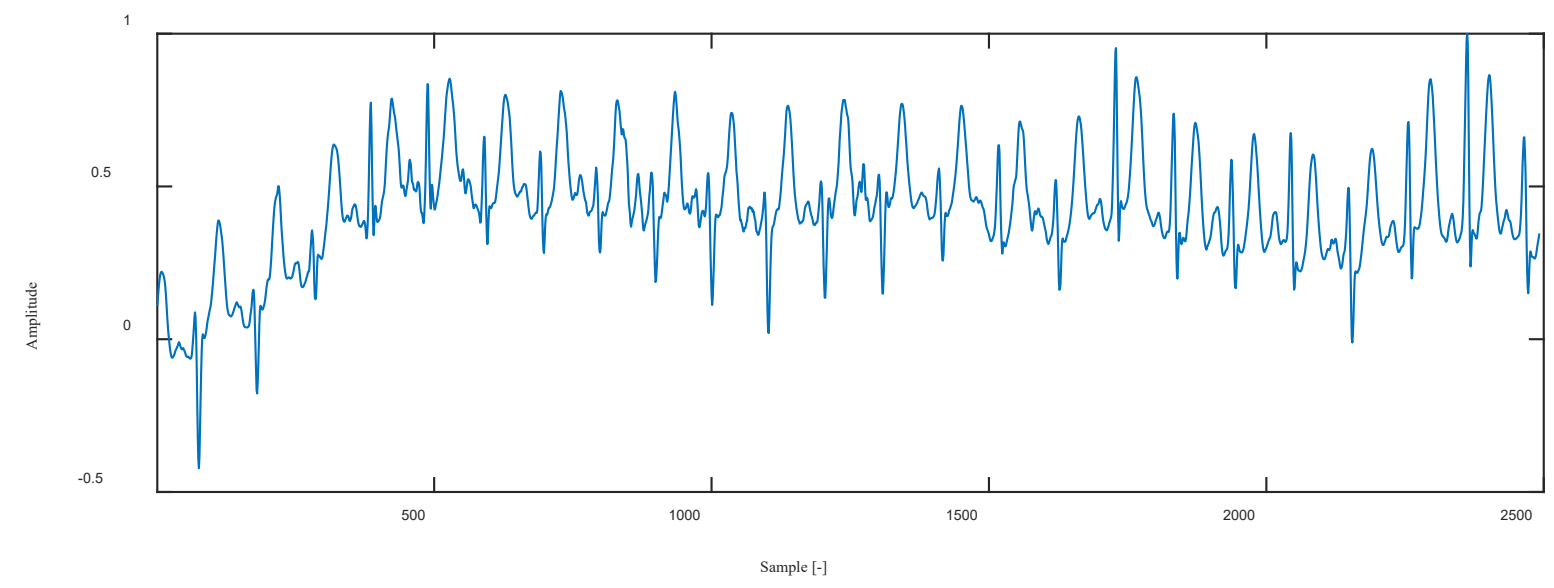

(c) - the filtered ECG by the proposed algorithm

Fig. 4. The performance of the adaptive filter under the jogging state, (a) - the real recorded ECG, (b) - the reference signal vector from the accelerometer, and (c) - the filtered ECG by using MEDNSS algorithm. 


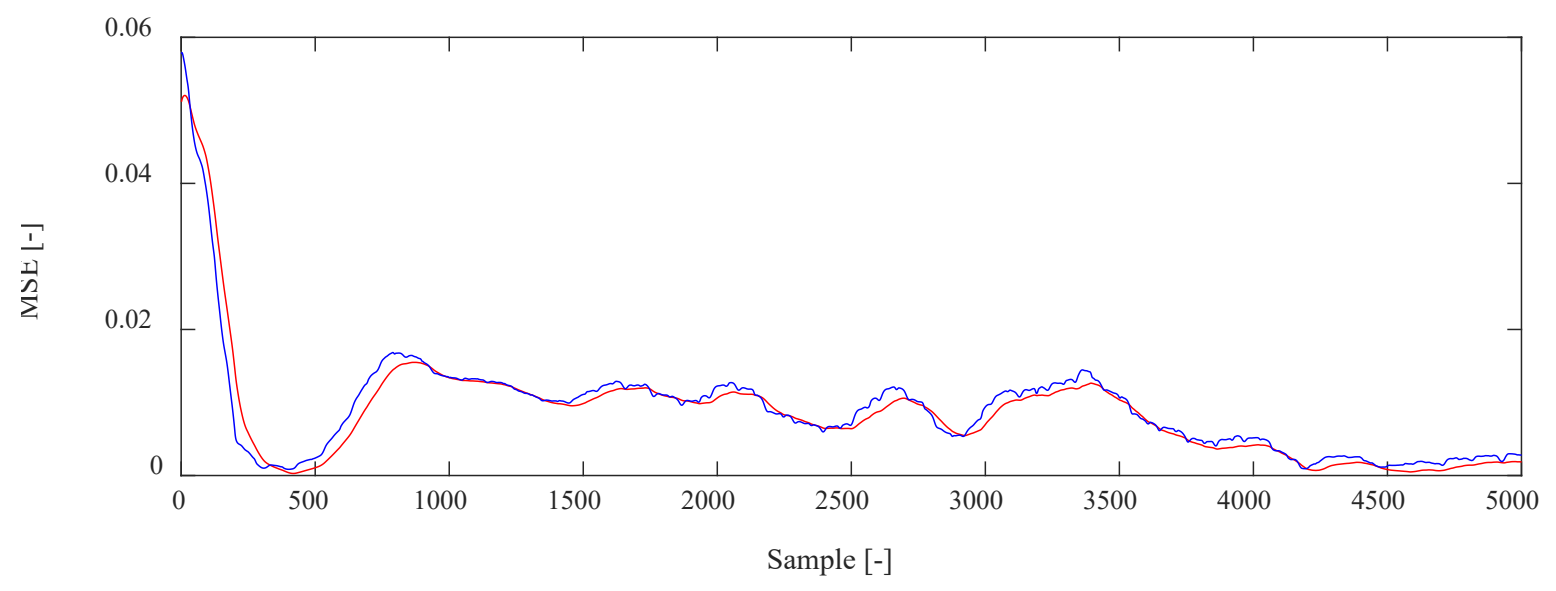

Fig. 5. The graph compares the EMSE value of two algorithms: blue line - EDNSS and red line - MEDNSS.

\section{Conclusion}

In this study, we describe the adaptive filtering technique to eliminate the motion artifacts by using the Modified Error Data Normalized Step-Size (MEDNSS) algorithm, which minimizes the Learning Curve based on the steepest descent strategy. The reference input signal for the adaptive filter is acquired from a 3-axis accelerometer. The filter performance of the algorithm is assessed on the stress ECG signals recorded with different scenarios from the University of Glasgow [10]. The results show that the proposed algorithm effectively improves the quality of the stress ECG signal.

\section{Acknowledgements}

This work has been supported by VNU University of Engineering and Technology under project number CN20.14.

\section{References}

[1] Nitish V. T., Yi-Sheng Z. - Applications of Adaptive Filtering to ECG Analysis: Noise Cancellation and Arrhythmia Detection, IEEE Trans. Biomed. Eng. 38 (8) (1991), 785-794.

[2] Michael H. C., Steven J. B., Michael J. S., Cynthia M. T., Thomas J. R., and Sidney C. S. - ACC/AHA Guidelines for Ambulatory Electrocardiography, Journal of the American College of Cardiology 34 (3) (1999) 913-948.

[3] Shafa-at A. S., Nauman R., Tahir Z. - Baseline Wander Removal From ECG Signal using State Space Recursive Least Squares (SSRLS) Adaptive Filter, 2nd
International Conference on Robotics and Artificial Intelligence, 2016, pp. 58-62.

[4] Yan Z., Fugui L., Zhigang Z., Dandan L., Xiaoyan Z., Jingyuan W. - Studies on application of support vector machine in diagnose of coronary heart disease, 6th International Conference on Electromagnetic Field Problems and Applications, 2012.

[5] Vinod K. P. - Adaptive filtering for baseline wander removal in ECG, Proceedings of the 10th IEEE International Conference on Information Technology and Applications in Biomedicine, 2010.

[6] Sung W. Y., Se D. M., Yong H. Y., Seungpyo L., Myoungho L. - Adaptive motion artifacts reduction using 3-axis accelerometer in E-textile ECG measurement system, J. Med. Syst. 32 (2) (2008) 101106.

[7] Tong D. A., Bartels K. A., and Honeyager K. S. Adaptive reduction of motion artifact in the electrocardiogram, Proceedings of the Second Joint EMBSBMES Conference, 2002, pp. 1403-1404.

[8] Mary Anne D. R., Luis G. S. - Adaptive noise cancelling of motion artifact in stress ECG signals using accelerometer, Proceedings of the Second Joint EMBSBMES Conference, 2002, pp. 1756-1757.

[9] Joonwan K., Alexander D. P. - Performance analysis of the adjusted step size NLMS algorithm, Proceedings of the 36th Southeastern Symposium on System Theory, 2004, pp. 467-471.

[10] Porr B, Howell L. - R-peak detector stress test with a new noisy ECG database reveals significant performance differences amongst popular detectors. BioRxiv 2019, pp. 1-2 